\title{
Clima y temperatura sub-superficial del mar en Bahía Culebra, Golfo de Papagayo, Costa Rica
}

\author{
Eric J. Alfaro ${ }^{1,2,3}$, Jorge Cortés ${ }^{1,4}$, Juan José Alvarado ${ }^{1,5}$, Carlos Jiménez ${ }^{1,6}$, Alberto León 7 , \\ Celeste Sánchez-Noguera ${ }^{1}$, Jaime Nivia-Ruiz ${ }^{1}$ \& Eleazar Ruiz ${ }^{1}$ \\ 1. Centro de Investigación en Ciencias del Mar y Limnología, \\ 2. Centro de Investigaciones Geofísicas \\ 3. Escuela de Física, Universidad de Costa Rica, 11501-2060 San José, Costa Rica; erick.alfaro@ucr.ac.cr. \\ 4. Escuela de Biología, Universidad de Costa Rica, 11501-2060 San José, Costa Rica \\ 5. Posgrado en Ciencias Marinas y Costeras, Universidad Autónoma de Baja California Sur, La Paz, México \\ 6. Oceanography Center, University of Cyprus, P.O. Box 20537, 1678 Nicosia, Cyprus \\ 7. 300 S 57th Ave., Hollywood, Florida 33023, EUA
}

$$
\text { Recibido 18-VIII-2011. Corregido 31-X-2011. Aceptado 15-II-2012. }
$$

\begin{abstract}
Climate and subsurface sea temperature in Bahía Culebra, Costa Rica. Bahía Culebra, Golfo de Papagayo, Costa Rica is a seasonal upwelling area. To determine the relationship of climate and the subsurface temperature variability at Bahía Culebra, we analyzed nine records of sea subsurface temperature from the Bay, continuously recorded from 1998 to 2010. The analysis characterized the annual cycle and explored the influence of different climate variability sources on the subsurface sea temperature and air temperature recorded in Bahía Culebra. Data from an automatic meteorological station in the bay were studied, obtaining the annual and daily cycle for air surface temperature and wind speed. Sea surface temperature (SST) trend from 1854 to 2011 was calculated from reanalysis for the region that coverts $9-11^{\circ} \mathrm{N}, 85-87^{\circ} \mathrm{W}$. Because of the positive SST trend identified in this region, results showed that annual and daily cycles in Bahía Culebra should be studied under a warming scenario since 1854, that is coherent with the global warming results and its climate variability is influenced by El Niño-Southern Oscillation (ENSO) in the Equatorial Pacific and by atmospheric forcing triggered by climate variability with Atlantic Ocean origin, because warm (cold) events in Bahía Culebra tend to occur in concordance with positive \& negative (negative \& positive) anomalies in Niño 3.4 (NAO) index. Rev. Biol. Trop. 60 (Suppl. 2): 159-171. Epub 2012 April 01.
\end{abstract}

Key words: Bahía Culebra, Costa Rica, sea temperature, air surface temperature, climatology, climate variability, ENSO, NAO, Central America, Golfo de Papagayo.

Bahía Culebra en Costa Rica, se ubica en el Golfo de Papagayo, en la vertiente del Pacífico al noroeste del país y presenta un eje norestesuroeste (Fig. 1). De acuerdo a la clasificación del Instituto Meteorológico Nacional de Costa Rica (IMN, http://www.imn.ac.cr, 22/06/2011) su régimen climático corresponde a la región del Pacífico Norte o seco (Fig. 2), ya que presenta un período de disminución de lluvias bastante marcado entre los meses de diciembre y marzo, seguido por dos periodos de máxima precipitación: el primero en mayo-junio y el segundo, mayor que el primero, en agosto-setiembre-octubre. Dichos máximos están separados por un periodo de disminución de la precipitación, normalmente observado en julio, llamado "veranillo" (también conocido como veranillo de San Juan o canícula). Los meses de abril y noviembre se consideran periodos de transición entre las temporadas secas y lluviosas y viceversa (Magaña et al. 1999, Taylor \& Alfaro 2005).

Bahía Culebra se encuentra en una zona donde la variabilidad climática producto del 

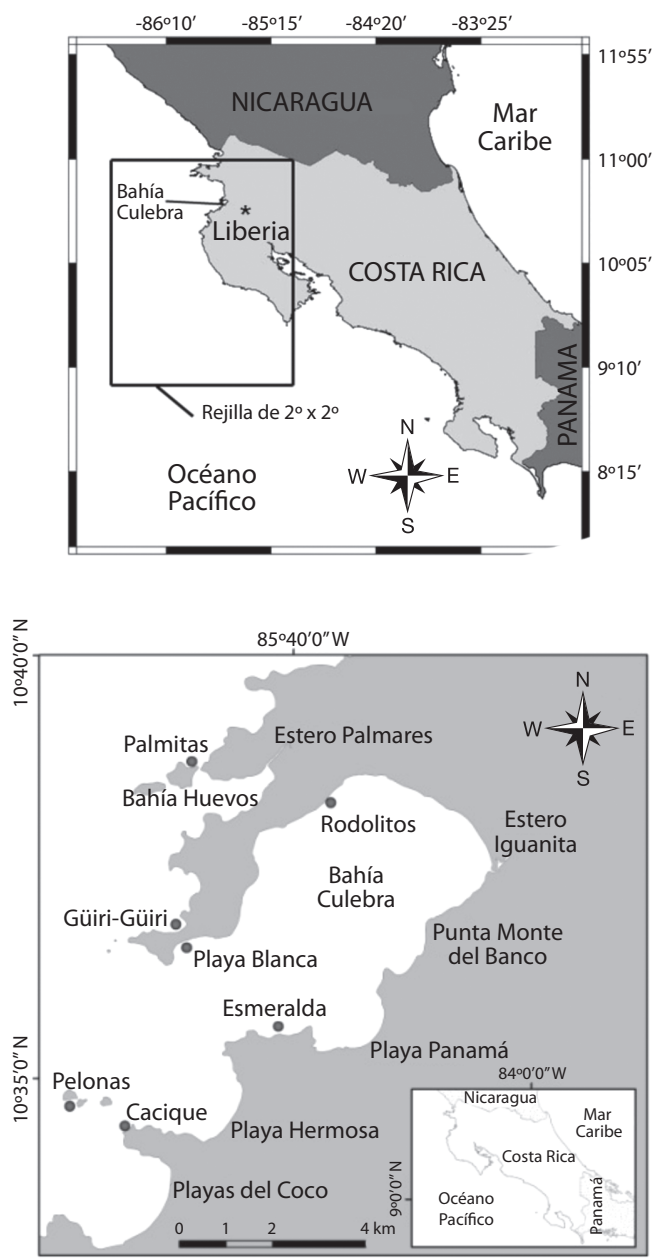

Fig. 1. a) Localización de Bahía Culebra, Costa Rica. El área del cuadro presentado en el mapa, corresponde al área usada para calcular el valor mensual de la temperatura superficial de mar del punto de rejilla centrado en $10^{\circ} \mathrm{N}$, $86^{\circ} \mathrm{W}$, de acuerdo a Smith et al. (2008). El asterisco representa la ubicación de la estación meteorológica de Liberia. b) Ubicación geográfica de las estaciones de temperatura subsuperficial del mar usadas en Bahía Culebra.

Fig. 1. a) Location of Bahía Culebra, Costa Rica. The square showed in the map is the area used to calculate the monthly sea surface temperature value for the grid point centered in $10^{\circ} \mathrm{N}, 86^{\circ} \mathrm{W}$, according to Smith et al. (2008). The asterisk represent the location of the Liberia meteorological station. b) Geographic distribution of the sea subsurface temperature stations used in Bahía Culebra.

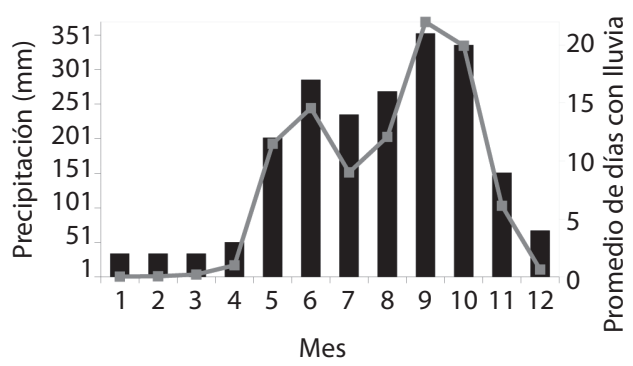

Fig. 2. Precipitación mensual acumulada promedio (línea gris con cuadros) y el promedio del número mensual de días en los cuales se registró precipitación (barras verticales negras), ambas variables observadas en la estación de Liberia, Costa Rica (10³5' N, 85³2'W, 80 msnm, 1977 2010; http://www.imn.ac.cr).

Fig. 2. Squared gray line is for the average of monthly accumulated precipitation and the vertical black bars are for the average of monthly rainy day occurrences. Data were observed in the station of Liberia, Costa Rica $\left(10^{\circ} 35^{\prime}\right.$ N, 85³2'W, 80 masl, 1977-2010; http://www.imn.ac.cr).

afloramiento costero (diciembre-abril) afecta el comportamiento de las temperaturas superficiales del mar (TSM), las cuales pueden llegar a descender hasta $17^{\circ} \mathrm{C}$ (Alfaro \& Cortés 2011). Así mismo, durante la época lluviosa (mayonoviembre) la TSM es más estable $\left(\sim 28^{\circ} \mathrm{C}\right)$ (Amador et al. 2006). Este cambio en los patrones de TSM puede tener efectos positivos y negativos sobre la biota presente en esta bahía (e.g. Jiménez et al. 2001).

Sobre la vertiente del Pacífico de Costa Rica, la magnitud del viento es normalmente más fuerte durante el invierno y primavera boreal (diciembre a mayo), con dirección predominante del este (vientos alisios) y disminuye durante el verano y otoño boreal, entre junio y noviembre (Alfaro 2002). Durante estas dos últimas estaciones climáticas del año también se observa la formación de sistemas mesoescalares de brisa marina, asociados a aguaceros vespertinos, acompañados algunas veces de tormentas eléctricas. Existen dos sistemas sinópticos transitorios dominantes sobre Costa 
Rica, durante el invierno-primavera son los frentes fríos que incursionan por el Caribe, los cuales refuerzan el viento con componente del este sobre el país. Durante el verano-otoño, los ciclones tropicales del Atlántico y del Caribe son los que favorecen la aparición de vientos con componente del oeste u "oestes sinópticos" (Muñoz et al. 2002).

Las temperaturas del aire mínimas registradas en la estación meteorológica de Liberia, ubicada $13 \mathrm{~km}$ al este de Bahía Culebra, presentan un máximo durante mayo-junio y un mínimo durante el invierno boreal, entre diciembre y febrero (Fig. 3). El máximo de la temperatura mínima coincide a su vez con el primer máximo de precipitación de la estación lluviosa. Por su parte las mayores temperaturas máximas se observan durante marzo-abril, previo al inicio de la temporada lluviosa y las menores durante setiembre-octubre, cuando se presenta la mayor cantidad de precipitación del ciclo anual, siendo abril el mes de la máxima temperatura media y octubre y noviembre los de menor temperatura media (Fig. 3).

De acuerdo con Amador et al. (2006), durante el invierno boreal, la incursión de masas de aire polar que se mueven hacia el sur y se adentran en el Mar Caribe, producen

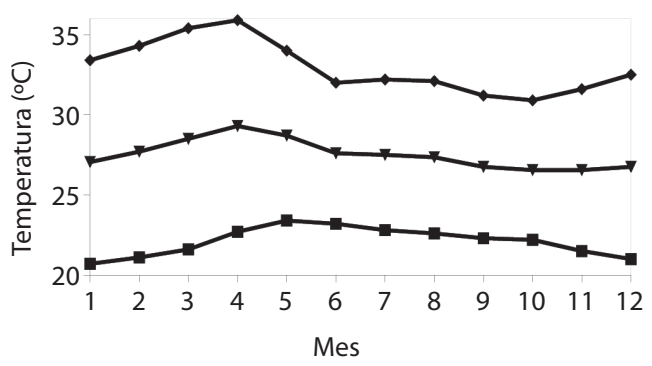

Fig. 3. Promedio mensual de las temperaturas máximas (línea con rombos) y mínimas (línea con cuadros) observadas en la estación de Liberia, Costa Rica, de 1977 a 2010. El promedio de ambas corresponde a la línea con triángulos.

Fig. 3. Diamond (squared) line is for the monthly average of maximum and minimum temperatures observed in Liberia, Costa Rica, from 1977 to 2010. The average of them is plotted in the line with triangles. fuertes gradientes de presión entre el Mar Caribe y el Pacífico Tropical del Este. Este viento con componente del este se canaliza a través de pasos de altura baja del sur de México y América Central. Uno de estos pasos se ubica entre las tierras bajas del centro de Nicaragua y el norte de Costa Rica. Los vientos producidos por esta canalización son llamados comúnmente como Papagayos o Corriente en Chorro de Papagayo (e.g. Amador et al. 2006), debido al golfo del mismo nombre ubicado en el Pacífico norte de Costa Rica y siendo Bahía Culebra parte de él. Según Amador et al. (2006), el viento en capas bajas de la atmósfera durante estos eventos puede alcanzar velocidades máximas de hasta $50 \mathrm{~ms}^{-1}$. Adicionalmente, Amador (2008) encontró que al analizar el ciclo anual de la Corriente en Chorro de Bajo Nivel del Caribe durante el invierno boreal, ésta presenta un máximo secundario, lo cual influye también en el reforzamiento del viento con componente del este sobre el norte de Costa Rica.

El objetivo de este trabajo es estudiar la climatología de los registros de temperatura superficial del mar (TSM) y sub-superficial (TSSM) en Bahía Culebra, y su relación con fuentes de variabilidad conocidas como $\mathrm{El}$ Niño-Oscilación del Sur (ENOS) y la Oscilación de Atlántico Norte (OAN). Este tipo de análisis es muy importante por dos aspectos. Primero, existen muy pocos estudios climáticos en las zonas costeras de Costa Rica y segundo, también son poco frecuentes los registros de TSSM en las regiones costeras que abarquen más de diez años, por lo que su análisis es prácticamente único en el país.

\section{MATERIALES Y MÉTODOS}

Al igual que en Alfaro y Cortés (2011), se usaron los registros de TSSM (Hobotemp ${ }^{\circledR}$, Onset Computer Corp.) de nueve estaciones en Bahía Culebra (Fig. 1b). Todos los sensores fueron ubicados en profundidades iguales o mayores a $3.5 \mathrm{~m}$. Estos sensores fueron programados para registrar el dato de la temperatura cada 30 minutos y fueron reemplazados cada mes desde 1998 hasta el 2010. Los registros 
de las estaciones de monitoreo en los arrecifes de Güiri Güiri intermedio en Ensenada Viradores del Norte, Rodolitos y Playa Blanca se empezaron a tomar en 1999, 2003 y 2004, respectivamente.

El procesamiento de los registros de TSSM fue similar al de Alfaro y Cortés (2011), donde primero se produjeron series de tiempo con resolución temporal horaria para cada una de las estaciones. Luego, los datos faltantes de los registros horarios se rellenaron con el promedio histórico de la serie correspondiente. Para filtrar señales con periodicidades menores a un mes, se filtraron las señales de alta frecuencia con una media móvil triangular de 744 datos (31 días aproximadamente) de acuerdo a Soley (1994). Después las series se normalizaron restando los valores mensuales del promedio mensual histórico de la serie y su posterior división por la desviación estándar mensual histórica (o sea, de todos los datos horarios del mes correspondiente presentes en la serie de tiempo). Lo anterior permite la comparación entre los eventos al eliminar la influencia del ciclo anual y entre las estaciones, al eliminar la variabilidad espacial. Para cada estación, se identificaron los meses cálidos y los meses fríos, como también aquellos periodos en los cuales las desviaciones estándar de las anomalías normalizadas fueron mayores o menores a $1.5 \mathrm{y}-1.5$ durante un mes determinado. Adicionalmente, se tomaron como eventos cálidos o fríos, sólo aquellos en los cuales estos hayan sido observados en al menos cuatro estaciones de monitoreo.

Se obtuvieron y analizaron los datos horarios de una estación meteorológica automática la cual funcionó en forma intermitente en Playa Panamá (10³5'13”N, 85³7’35”'W, Fig. 1b), entre los años 1999 y 2005. Debido a la intermitencia de dichos registros, luego del análisis exploratorio y de estudiar la meta-información asociada, se determinó que estos no eran aptos para su uso como series de tiempo y se procedieron a calcular únicamente los ciclos anuales y diarios de las series de temperatura media del aire, la magnitud del viento y los ciclos anuales de las temperaturas máximas y mínimas.
Se usó el registro mensual de la temperatura superficial del mar (TSM) correspondiente al punto centrado en $10^{\circ} \mathrm{N}, 86^{\circ} \mathrm{W}$ (Fig. 1a), de una rejilla de $2^{\circ} \times 2^{\circ}$ del conjunto de datos elaborado por Smith et al. (2008) disponible en el repositorio del Instituto Internacional para la Investigación del Clima y la Sociedad (http://iridl.ldeo.columbia.edu/, última visita 27/06/2011), para el período de enero de 1854 a mayo de 2011.

Por último, los índices de variabilidad climática asociados a El Niño-Oscilación del Sur (ENOS), Niño 3.4, y a la Oscilación del Atlántico Norte (OAN, Hurrell 2005) se obtuvieron del repositorio de índices climáticos de la NOAA (http://www.cpc.ncep.noaa.gov/data/ indices/, última visita 02/03/2011). Posteriormente se utilizó un análisis de tabla de contingencia (Alfaro et al. 2003) y se calcularon las probabilidades empíricas absolutas y relativas para los eventos cálidos y fríos en Bahía Culebra dada una observación positiva o negativa en los índices climáticos.

\section{RESULTADOS}

La TSM en esa región presenta un máximo en mayo y un mínimo en enero (Fig. 4). El análisis de la TSM mostró que Bahía Culebra se encuentra en una región en la cual se observa un calentamiento entre enero de 1854 y mayo de 2011. La tendencia lineal asociada a la serie

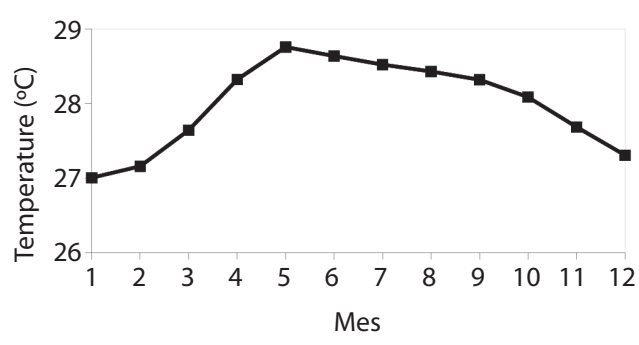

Fig. 4. Promedio mensual de la temperatura superficial del mar para la región mostrada en la Fig. 1, de acuerdo a Smith et al. (2008), de enero de 1854 a mayo de 2011.

Fig. 4. Monthly average for the sea surface temperature measured in the area showed in Fig. 1, according to Smith et al. (2008), from January, 1854 to May, 2011. 
de tiempo fue de $0.0013{ }^{\circ} \mathrm{C} / \mathrm{mes}$, significativa a más del $99 \%$ (Fig. 5) y el promedio de la serie fue de $27.9{ }^{\circ} \mathrm{C}$. Dicha tendencia se enmarca dentro del escenario del calentamiento global descrito por Bindoff et al. (2007) y Trenberth et al. (2007).

Las magnitudes máximas del viento entre 1999 y 2002 se observaron de enero a abril, en horas de la tarde (Fig. 6). En julio se observó un máximo secundario, el cual se asocia al incremento de la magnitud del viento zonal sobre la región por la Corriente en Chorro de Bajo Nivel del Caribe, que concuerda con el veranillo (Magaña et al. 1999; Amador 2008). El resultado mostrado en la Figura 6, coincide parcialmente con los resultados obtenidos en los estudios realizados por Wesson-Acuña (2005) y Wesson-Vizcaíno (2006). Estos autores tomaron mediciones intermitentes del viento superficial entre diciembre de 2003 y marzo de 2006, con un anemómetro a una altura de $5.5 \mathrm{~m}$ sobre el suelo en un sitio localizado a $10^{\circ} 38^{\prime} 35^{\prime \prime} \mathrm{N}$ y 85³9'08'W (Wesson-Vizcaíno 2006). Ellos encontraron que la dirección predominante del viento fue del noreste y del este, principalmente durante el invierno boreal, con magnitudes mayores a $6 \mathrm{~m} / \mathrm{s}$. Al analizar las horas del día

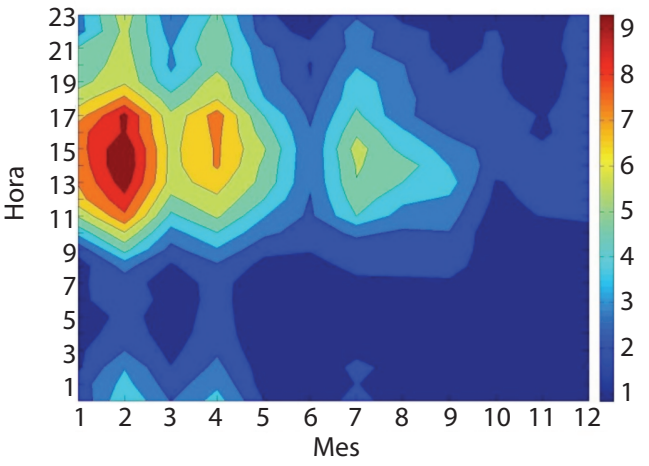

Fig. 6. Ciclo anual (eje x) y diario (eje y) de los datos de la estación automática ubicada en Playa Panamá, Bahía Culebra (10³5'13”'N, 85³7'35' W) entre los años 1999 y 2002 para la rapidez del viento $(\mathrm{m} / \mathrm{s})$.

Fig. 6. Annual (x axis) and daily (y axis) cycle of the automatic weather station data at Playa Panama, Bahía Culebra (10³5'13'”N, 85³7'35'W) from 1999 to 2002 for wind velocity $(\mathrm{m} / \mathrm{s})$.

en donde se presentaban los máximos de la magnitud del viento, notaron que generalmente ocurrían entre las 8 y las 16 horas.

Los valores máximos de la temperatura media del aire entre 1999 y 2002 se observaron en febrero y marzo, también en horas de la tarde (Fig. 7). Las temperaturas vespertinas

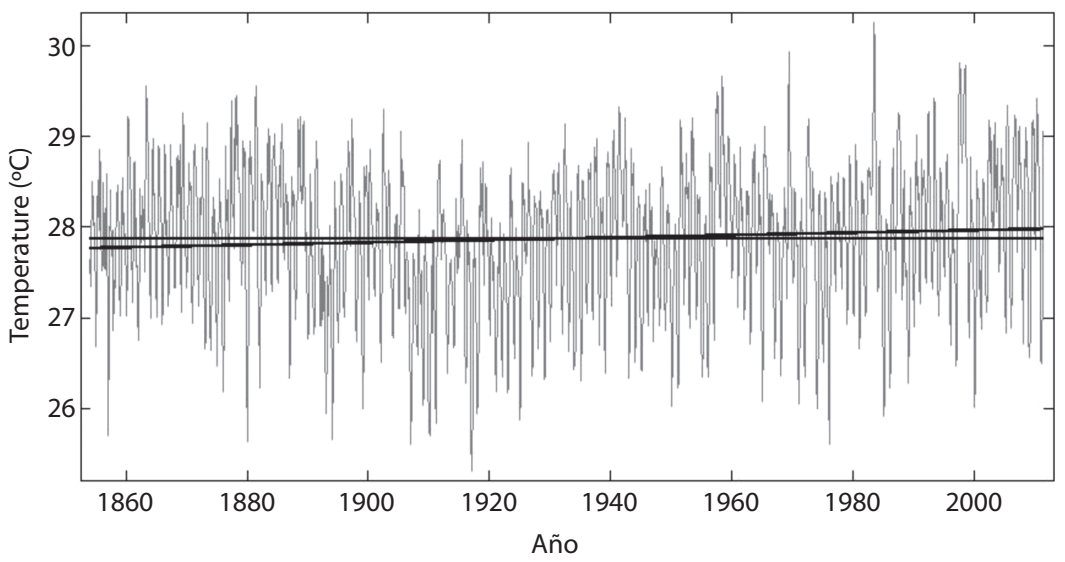

Fig. 5. La línea gris corresponde a la serie mensual de la temperatura superficial del mar para el área mostrada en la Fig. 1, de acuerdo a Smith et al. (2008). La línea horizontal corresponde al promedio de la serie de $27.9^{\circ} \mathrm{C}$. La tendencia lineal fue de $0.0013^{\circ} \mathrm{C} / \mathrm{mes}$, significativa a más del $99 \%$, para el periodo de enero de 1854 a mayo de 2011 (línea negra continua). Fig. 5. Gray line is for the monthly sea surface temperatures from the area showed in the Fig. 1, according to Smith $e t$ al. (2008). The horizontal line correspond the series average of $27.9^{\circ} \mathrm{C}$. Linear trend (solid black line) is $0.0013^{\circ} \mathrm{C} / \mathrm{month}$, with a statistical significance greater the 99\%, measured from January, 1854 to May, 2011. 


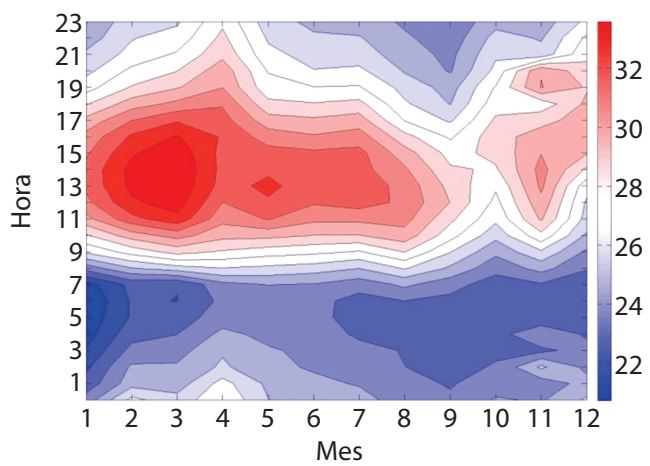

Fig. 7. Ciclo anual (eje x) y diario (eje y) de los datos de la estación automática ubicada en Playa Panamá, Bahía Culebra (10³5'13”'N, 85³7'35'W) entre los años 1999 y 2002 para la temperatura superficial del aire $\left({ }^{\circ} \mathrm{C}\right)$.

Fig. 7. Annual (x axis) and daily (y axis) cycle of the automatic weather station data at Playa Panama, Bahía Culebra $\left(10^{\circ} 35^{\prime} 13\right.$ 'N, $\left.85^{\circ} 37^{\prime} 35^{\prime \prime} \mathrm{W}\right)$ from 1999 to 2002 for air surface temperature $\left({ }^{\circ} \mathrm{C}\right)$.

disminuyen en setiembre-octubre, meses en los cuales se observan también las mayores precipitaciones (Fig. 2). Los valores mínimos de la temperatura media para esos mismos años, correspondieron a las horas de la madrugada del mes de enero, lo cual coincide con el mes del mínimo de la TSM (Fig. 4) y de poca cobertura nubosa en la región (Alfaro 2000, 2002). Las temperaturas máximas mayores corresponden a marzo-abril, previo al inicio de la estación lluviosa y sus valores mínimos se presentan en setiembre-octubre (Fig. 8), meses en los cuales la precipitación es máxima en la región (Fig. 2). La temperatura mínima alcanza su máximo en el mes de junio, que coincide con el primer máximo de la estación lluviosa y su mínimo en enero, al igual que la TSM. El promedio de ambas curvas muestra que a lo largo del ciclo anual el valor máximo se da en abril y el mínimo en setiembre.

Los resultados asociados a la variabilidad de los diferentes registros de temperatura se presentan en el Cuadro 1. Los valores asociados a la temperatura media del aire en ese cuadro fueron calculados a partir de valores horarios, los de las temperaturas máximas y mínimas a partir de datos diarios y los de la

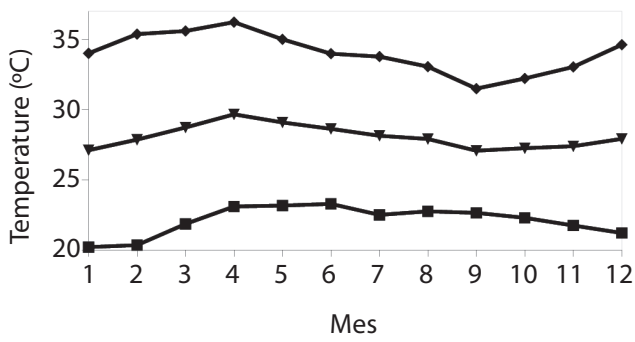

Fig. 8. La línea con rombos representa el promedio mensual de las temperaturas máximas del aire observadas en la estación de Bahía Culebra, Costa Rica y la línea con cuadros el correspondiente para las temperaturas mínimas. El promedio de ambas corresponde a la línea con triángulos.

Fig. 8. Diamond (squared) line is for the monthly average of maximum and minimum air temperatures observed in Bahía Culebra, Costa Rica. The average of them is plotted in the line with triangles.

TSM de datos mensuales. Nótese que el bimestre de mayor variabilidad de la temperatura media fue el de marzo-abril y el de la mínima el de setiembre-octubre. La máxima variabilidad en los registros de la temperatura máxima se observó durante mayo-junio, posterior al de la temperatura media y su mínima variabilidad se encontró durante el invierno boreal en enero-febrero. Agosto-setiembre mostró la mínima variabilidad en la temperatura mínima y febrero la máxima. El trimestre de agostosetiembre-octubre, asociado con las máximas precipitaciones, mostró la menor variabilidad de la TSM, siendo enero-febrero-marzo el de la máxima variabilidad.

En promedio, los valores mínimos de la TSSM, se alcanzan durante febrero-marzo, contrario a lo observado en la Figura 6 para la temperatura superficial del aire; y los máximos en junio y agosto (Cuadro 2). Individualmente, los valores máximos de la TSSM se registraron en agosto para la estación de Rodolitos y los mínimos en febrero para la estación de Güiri Güiri profundo. Al analizar los registros de la variabilidad (Cuadro 3), se observa que en promedio el bimestre de mínima variabilidad fue julio-agosto y el de máxima variabilidad 
CUADRO 1

Desviación estándar de los registros horarios de la temperatura superficial del aire $\left({ }^{\circ} \mathrm{C}\right)$, y de los registros diarios de las temperaturas máximas y mínimas en Bahía Culebra. La última columna corresponde a los registros mensuales de la

Temperatura Superficial del Mar de la región mostrada en la Figura 1. Los valores en negrita corresponden a los máximos y mínimos de cada columna

\section{TABLE 1}

Standard deviation for the hourly record of air surface temperature $\left({ }^{\circ} \mathrm{C}\right)$, the daily records of maximum and minimum temperatures. These three observed in Bahía Culebra. Last column is for the monthly record of sea surface temperature shown in Figure 1. Bold values in every column correspond with the maximum and minimum

\begin{tabular}{ccccc} 
Mes & $\begin{array}{c}\text { Temperatura } \\
\text { media }\end{array}$ & $\begin{array}{c}\text { Temperatura } \\
\text { máxima }\end{array}$ & $\begin{array}{c}\text { Temperatura } \\
\text { mínima }\end{array}$ & $\begin{array}{c}\text { Temperatura } \\
\text { Superficial del Mar }\end{array}$ \\
1 & 3.92 & 1.51 & 1.35 & 0.64 \\
2 & 4.09 & $\mathbf{1 . 3 4}$ & 1.53 & 0.64 \\
3 & $\mathbf{4 . 1 7}$ & 1.64 & 1.44 & $\mathbf{0 . 6 5}$ \\
4 & 4.13 & 1.74 & 1.17 & 0.58 \\
5 & 3.48 & $\mathbf{2 . 6 3}$ & 0.95 & 0.54 \\
6 & 3.27 & 2.27 & 1.10 & 0.51 \\
7 & 3.57 & 1.80 & 0.78 & 0.49 \\
8 & 3.30 & 1.86 & $\mathbf{0 . 7 2}$ & 0.46 \\
9 & $\mathbf{2 . 6 9}$ & 2.12 & 0.68 & $\mathbf{0 . 4 5}$ \\
10 & 3.27 & 2.25 & 0.81 & 0.46 \\
12 & 3.66 & 1.98 & $\mathbf{1 . 5 7}$ & 0.51 \\
\hline
\end{tabular}

\section{CUADRO 2}

Valores medios mensuales de la temperatura subsuperficial del mar $\left({ }^{\circ} \mathrm{C}\right)$ en Bahía Culebra. Los valores en negrita corresponden a los máximos y mínimos de cada columna

TABLE 2

Monthly mean values of sea subsurface temperature $\left({ }^{\circ} \mathrm{C}\right)$ in Bahia Culebra. Bold values in every column correspond with the maximum and minimum

\begin{tabular}{cccccccccc} 
Mes & Palmitas & $\begin{array}{c}\text { Güiri Güiri } \\
\text { profundo }\end{array}$ & $\begin{array}{c}\text { Güiri Güiri } \\
\text { intermedio }\end{array}$ & $\begin{array}{c}\text { Güiri Güiri } \\
\text { somero }\end{array}$ & $\begin{array}{c}\text { Playa } \\
\text { Blanca }\end{array}$ & Rodolitos & Esmeralda & Cacique & Pelonas \\
1 & 26.33 & 25.82 & 26.07 & 26.50 & 27.21 & 27.19 & 26.79 & 26.48 & 26.49 \\
2 & $\mathbf{2 5 . 5 8}$ & $\mathbf{2 4 . 8 5}$ & $\mathbf{2 5 . 2 1}$ & $\mathbf{2 5 . 4 2}$ & 26.60 & $\mathbf{2 6 . 8 2}$ & $\mathbf{2 5 . 4 2}$ & $\mathbf{2 5 . 8 3}$ & $\mathbf{2 5 . 6 1}$ \\
3 & 25.97 & 25.28 & 25.72 & 26.35 & $\mathbf{2 6 . 4 4}$ & 26.91 & 25.55 & 26.44 & 25.87 \\
4 & 26.99 & 26.45 & 26.93 & 26.93 & 27.47 & 27.89 & 26.78 & 27.23 & 27.04 \\
5 & 28.66 & 28.21 & 28.58 & 28.50 & 28.42 & 28.90 & 28.08 & 28.29 & 28.44 \\
6 & 28.74 & 28.16 & 28.51 & 28.66 & $\mathbf{2 8 . 6 7}$ & 29.01 & 28.37 & 28.91 & 28.39 \\
7 & 28.55 & 28.18 & 28.49 & 28.37 & 28.61 & 29.06 & 28.29 & 28.36 & 28.34 \\
8 & $\mathbf{2 8 . 8 4}$ & $\mathbf{2 8 . 3 5}$ & $\mathbf{2 8 . 7 3}$ & $\mathbf{2 8 . 8 1}$ & 26.06 & $\mathbf{2 9 . 2 6}$ & $\mathbf{2 8 . 7 8}$ & $\mathbf{2 8 . 5 6}$ & $\mathbf{2 8 . 6 0}$ \\
9 & 28.50 & 28.30 & 28.69 & $\mathbf{2 8 . 8 1}$ & 28.42 & 29.11 & 28.68 & 28.32 & 28.49 \\
10 & 27.73 & 27.33 & 27.79 & 27.94 & 28.11 & 28.65 & 27.78 & 27.68 & 27.88 \\
11 & 26.74 & 26.78 & 26.70 & 27.33 & 27.13 & 27.84 & 27.13 & 27.06 & 27.12 \\
12 & 26.61 & 26.41 & 26.60 & 27.02 & 27.23 & 27.48 & 26.99 & 26.89 & 26.88 \\
\hline
\end{tabular}


CUADRO 3

Valores medios mensuales de la desviación estándar de la temperatura subsuperficial del mar $\left({ }^{\circ} \mathrm{C}\right)$ en Bahía Culebra. Los valores en negrita corresponden a los máximos y mínimos de cada columna

TABLE 3

Monthly mean standard deviation values of sea subsurface temperature $\left({ }^{\circ} \mathrm{C}\right)$ in Bahia Culebra. Bold values in every column correspond with the maximum and minimum

$\begin{array}{cccccccccc}\text { Mes } & \text { Palmitas } & \begin{array}{c}\text { Güiri Güiri } \\ \text { profundo }\end{array} & \begin{array}{c}\text { Güiri Güiri } \\ \text { intermedio }\end{array} & \begin{array}{c}\text { Güiri Güiri } \\ \text { somero }\end{array} & \begin{array}{c}\text { Playa Blanca } \\ \text { Rodolitos }\end{array} & \text { Esmeralda } & \text { Cacique } & \text { Pelonas } \\ 1 & 1.28 & 1.26 & 1.14 & 1.38 & 0.56 & 0.85 & 0.99 & 1.28 & 1.17 \\ 2 & 1.56 & 1.63 & 1.60 & 1.40 & 1.31 & \mathbf{1 . 7 7} & 1.68 & 1.30 & 1.49 \\ 3 & \mathbf{1 . 6 8} & \mathbf{1 . 7 6} & \mathbf{1 . 7 0} & \mathbf{1 . 4 8} & \mathbf{1 . 4 3} & 1.62 & 1.70 & \mathbf{1 . 5 6} & \mathbf{1 . 7 3} \\ 4 & 1.01 & 1.16 & 1.17 & 0.98 & 0.69 & 0.68 & 1.18 & 1.47 & 1.42 \\ 5 & 0.84 & 1.01 & 0.92 & 0.90 & 0.78 & 0.79 & \mathbf{1 . 7 6} & 0.89 & 0.92 \\ 6 & 0.72 & 0.82 & 0.78 & 0.65 & 0.60 & 0.60 & 1.11 & 1.80 & 0.72 \\ 7 & 0.61 & 0.60 & 0.50 & 0.52 & 1.27 & \mathbf{0 . 4 0} & 0.66 & 0.63 & \mathbf{0 . 5 8} \\ 8 & \mathbf{0 . 4 9} & \mathbf{0 . 7 2} & \mathbf{0 . 4 8} & \mathbf{0 . 5 1} & 0.93 & 0.51 & \mathbf{0 . 6 1} & \mathbf{0 . 4 9} & 0.61 \\ 9 & 0.77 & 0.82 & 0.64 & 0.70 & 1.35 & 0.85 & 0.81 & 0.70 & 0.70 \\ 10 & 1.05 & 1.29 & 1.11 & 1.07 & 0.84 & 0.71 & 1.20 & 0.93 & 0.66 \\ 11 & 1.43 & 1.09 & 1.39 & 1.01 & \mathbf{0 . 7 5} & 0.90 & 1.01 & 1.37 & 0.94 \\ 12 & 1.19 & 1.17 & 1.10 & 1.14 & 0.76 & 0.71 & 1.02 & 1.20 & 0.95\end{array}$

fue febrero-marzo. Individualmente, junio en la estación de Cacique presentó el valor máximo y julio en Rodolitos el mínimo. La Figura 9 muestra el ciclo anual para las temperaturas máximas, medias y mínimas en la estación de Güiri Güiri intermedio, la cual presentó muy pocos meses sin datos mensuales (nueve meses). Se observó un mínimo durante el mes de febrero para las tres variables presentadas en la Fig. 9. Al igual que la TSM, la temperatura máxima en la estación de Güiri Güiri intermedio, alcanzó su máximo en el mes de mayo, sin embargo los máximos asociados a la temperatura media y mínima, se observaron durante el mes de agosto. Cabe destacar que al analizar el ciclo diurno de los registros de TSSM, se encontró que éste es muy pequeño, por lo que la principal variabilidad de la TSSM en todos los sitios de medición está asociada al ciclo anual.

Catorce eventos cálidos fueron encontrados, todos en concordancia con anomalías positivas de la TSM en la región mostrada en el Figura 1 (Cuadro 4). Por otra parte, se identificaron 26 eventos fríos, los cuales se muestran

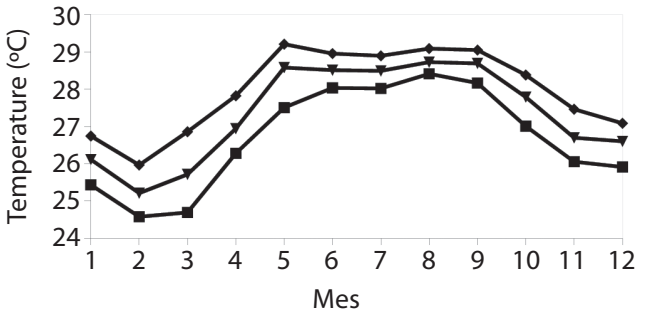

Fig. 9. La línea con rombos representa el promedio mensual de las temperaturas máximas subsuperficiales observadas en la estación de Guiri Güiri intermedio, Bahía Culebra, Costa Rica y la línea con cuadros el correspondiente para las temperaturas mínimas. La temperatura media corresponde a la línea con triángulos.

Fig. 9. Diamond (squared) line is for the monthly average of maximum and minimum subsurface temperatures observed in Güiri Güiri intermedio, Bahía Culebra, Costa Rica. The mean temperature is plotted in the line with triangles.

en el Cuadro 5, de los cuales solamente el 54\% de los mismos coincidieron con anomalías negativas de la TSM en dicha región.

Con el fin de explorar el escenario climático asociado a los eventos cálidos y fríos (Cuadros $4,5)$ con fuentes de variabilidad conocidas 


\section{CUADRO 4}

Años y meses asociados (primera y segunda columna) con anomalías normalizadas mayores a 1.5 en al menos 4 estaciones de registro de la TSSM en Bahía Culebra (tercera columna). Los valores entre paréntesis corresponden al dato máximo registrado en cada estación. Se incluyen también los valores de las anomalías de la TSM mostrada en la Fig. 4 (cuarta columna). Las abreviaciones usadas son Pa (Palmitas), GP (Güiri Güiri profundo), GI (Güiri Güiri intermedio),

GS (Güiri Güiri somero), PB (Playa Blanca), Ro (Rodolitos), Es (Esmeralda), Ca (Cacique) e IP (Islas Pelonas)

TABLE 4

Years and months associated (first and second columns) with standardized anomalies greater than 1.5 in at least four SSST station records from Bahía Culebra (third column). Values in parenthesis are the maximum recorded in every station. Last column are the SST anomalies from Fig. 4. The acronymus used are Pa (Palmitas), GP (Güiri Güiri profundo), GI (Güiri

Güiri intermedio), GS (Güiri Güiri somero), PB (Playa Blanca), Ro (Rodolitos), Es (Esmeralda), Ca (Cacique) and IP (Islas Pelonas)

\begin{tabular}{cclc} 
Año & Mes & \multicolumn{1}{c}{ Estaciones(desviación estándar) } & TSM \\
1998 & 2 & $\mathrm{GP}(1.93), \mathrm{GI}(1.88), \mathrm{Es}(1.69), \mathrm{IP}(1.74)$ & 1.94 \\
1998 & 3 & $\mathrm{GP}(1.80), \mathrm{GI}(1.78), \mathrm{Es}(1.88), \mathrm{IP}(1.67)$ & 1.86 \\
1998 & 4 & $\mathrm{~Pa}(1.84), \mathrm{GP}(1.85), \mathrm{GI}(1.68) \mathrm{Es}(1.82)$ & 1.39 \\
1998 & 7 & $\mathrm{~Pa}(1.98), \mathrm{GP}(2.10), \mathrm{GI}(2.13), \mathrm{Es}(2.04), \mathrm{Ca}(1.82), \mathrm{IP}(2.16)$, & 0.68 \\
1998 & 8 & $\mathrm{~Pa}(1.70), \mathrm{GP}(1.81), \mathrm{GI}(2.15), \mathrm{Es}(1.74), \mathrm{Ca}(2.16), \mathrm{IP}(1.96)$ & 0.82 \\
1998 & 9 & $\mathrm{~Pa}(1.53), \mathrm{GP}(1.53), \mathrm{GI}(1.58), \mathrm{Ca}(1.86), \mathrm{IP}(1.83)$ & 0.71 \\
2003 & 4 & $\mathrm{~Pa}(1.85), \mathrm{GP}(1.77), \mathrm{GI}(2.09), \mathrm{GS}(2.65), \mathrm{Es}(1.70), \mathrm{Ca}(1.54)$ & 0.51 \\
2005 & 3 & $\mathrm{~Pa}(1.72), \mathrm{GP}(1.52), \mathrm{GI}(1.66), \mathrm{GS}(1.76), \mathrm{Ro}(1.57), \mathrm{IP}(1.74)$ & 0.71 \\
2005 & 4 & $\mathrm{~Pa}(1.86), \mathrm{PB}(2.10), \mathrm{GP}(1.62), \mathrm{GS}(1.89), \mathrm{Ro}(1.56), \mathrm{IP}(4.16)$ & 0.66 \\
2006 & 7 & $\mathrm{~Pa}(1.53), \mathrm{GP}(1.52), \mathrm{GS}(1.82), \mathrm{Ro}(1.96)$, & 1.07 \\
2006 & 11 & $\mathrm{~Pa}(1.63), \mathrm{GP}(1.80), \mathrm{GI}(1.61), \mathrm{GS}(1.66), \mathrm{Ro}(1.55), \mathrm{Es}(1.96), \mathrm{IP}(1.78)$ & 0.83 \\
2007 & 2 & $\mathrm{~Pa}(1.65), \mathrm{GP}(1.84), \mathrm{GI}(1.70), \mathrm{GS}(1.88), \mathrm{Es}(1.69), \mathrm{IP}(1.54)$ & 0.64 \\
2009 & 6 & $\mathrm{~Pa}(1.88), \mathrm{GP}(1.79), \mathrm{GI}(1.85), \mathrm{GS}(2.03), \mathrm{Ro}(2.02), \mathrm{IP}(1.79)$ & 0.86 \\
\hline
\end{tabular}

sobre América Central, se calculó la tabla de contingencia para los eventos cálidos, dada una anomalía positiva o negativa en ese mes en los índices de Niño 3.4, asociado con el ENSO y el de la Oscilación del Atlántico Norte (OAN), asociado con la variabilidad interanual en el Atlántico. El 58\% de los eventos cálidos se presentaron bajo condiciones de anomalías positivas en el Niño 3.4 y negativas en la OAN (Cuadro 6). Adicionalmente, el $54 \%$ de los eventos fríos se presentaron en concordancia con anomalías negativas en el Niño 3.4 y positivas en la OAN.

\section{DISCUSIÓN}

Un calentamiento en el Pacífico Ecuatorial Tropical podría influenciar la aparición de condiciones cálidas en Bahía Culebra (Cuadro 6), hecho que se vería potenciado con el debilitamiento de los vientos alisios sobre América Central bajo condiciones de anomalías negativas de la OAN (Hurrell 2005). La ocurrencia de un evento cálido en Bahía Culebra en concordancia con anomalías negativas en el Niño 3.4 y positivas en la OAN es muy poco probable, ya que su probabilidad empírica condicional es cero (Cuadro 6). El estudio de estos eventos cálidos en Bahía Culebra reviste especial importancia para las poblaciones de coral existentes, ya que se han reportado blanqueamientos y mortalidad de los mismos en la región durante la ocurrencia de algunos de estos eventos cálidos (e.g. Jiménez et al. 2001). Por otro lado y en forma opuesta al escenario anterior, un evento frío en la Bahía puede darse por la influencia de un enfriamiento en el Pacífico Ecuatorial Tropical, el cual se vería reforzado por un incremento de la magnitud del viento 


\section{CUADRO 5}

Años y meses asociados (primera y segunda columna) con anomalías normalizadas menores a -1.5 en al menos 4 estaciones de registro de la TSSM en Bahía Culebra (tercera columna). Los valores entre paréntesis corresponden al dato mínimo registrado en cada estación. Se incluyen también los valores de las anomalías de la TSM mostrada en la Fig. 4 (cuarta columna). Las abreviaciones usadas son Pa (Palmitas), GP (Güiri Güiri profundo), GI (Güiri Güiri intermedio),

GS (Güiri Güiri somero), PB (Playa Blanca), Ro (Rodolitos), Es (Esmeralda), Ca (Cacique) e IP (Islas Pelonas)

TABLE 5

Years and months associated (first and second columns) with standardized anomalies less than -1.5 in at least four SSST station records from Bahía Culebra (third column). Values in parenthesis are the maximum recorded in every station. Last column are the SST anomalies from Fig. 4. The acronymus used are Pa (Palmitas), GP (Güiri Güiri profundo), GI (Güiri

Güiri intermedio), GS (Güiri Güiri somero), PB (Playa Blanca), Ro (Rodolitos), Es (Esmeralda), Ca (Cacique) and IP (Islas Pelonas)

\begin{tabular}{|c|c|c|c|}
\hline Año & Mes & Estaciones(desviación estándar) & TSM \\
\hline 1999 & 9 & $\mathrm{~Pa}(-2.96), \mathrm{GP}(-3.96), \mathrm{GI}(-4.01), \mathrm{GS}(-3.70), \mathrm{Es}(-3.70), \mathrm{Ca}(-2.72)$, & -0.42 \\
\hline 1999 & 10 & $\mathrm{~Pa}(-2.62), \mathrm{GP}(-2.70), \mathrm{GI}(-2.53), \mathrm{GS}(-2.59) \mathrm{Es}(-2.75), \mathrm{Ca}(-2.64)$ & -0.55 \\
\hline 1999 & 11 & $\mathrm{~Pa}(-1.68), \mathrm{GP}(-2.37), \mathrm{GS}(-2.81), \mathrm{Es}(-2.20), \mathrm{Ca}(-1.67), \mathrm{IP}(-2.22)$ & -0.44 \\
\hline 1999 & 12 & $\mathrm{~Pa}(-2.22), \mathrm{GP}(-2.26), \mathrm{GI}(-2.26), \mathrm{GS}(-2.67), \mathrm{Es}(-2.84), \mathrm{Ca}(-2.24), \mathrm{IP}(-2.58)$ & -0.60 \\
\hline 2000 & 1 & $\mathrm{~Pa}(-2.30), \mathrm{GP}(-2.15), \mathrm{GI}(-2.27), \mathrm{GS}(-2.29), \mathrm{Es}(-3.19), \mathrm{Ca}(-2.16), \mathrm{IP}(-2.31)$ & -0.92 \\
\hline 2000 & 8 & $\mathrm{~Pa}(-2.00), \mathrm{GI}(-1.89), \mathrm{GS}(-2.23), \mathrm{Es}(-1.67), \mathrm{Ca}(-1.98), \mathrm{IP}(-1.97)$ & -0.02 \\
\hline 2001 & 1 & $\mathrm{~Pa}(-3.04), \mathrm{GP}(-3.07), \mathrm{GI}(-3.30), \mathrm{GS}(-2.88), \mathrm{Es}(-1.86), \mathrm{Ca}(-2.94), \mathrm{IP}(-3.44)$ & 0.19 \\
\hline 2001 & 2 & $\mathrm{~Pa}(-2.06), \mathrm{GP}(-1.84), \mathrm{GI}(-2.07), \mathrm{GS}(-2.11), \mathrm{Es}(-1.93), \mathrm{Ca}(-2.40), \mathrm{IP}(-2.48)$ & -0.19 \\
\hline 2001 & 4 & $\mathrm{~Pa}(-1.92), \mathrm{GP}(-1.70), \mathrm{GI}(-1.82), \mathrm{GS}(-1.63), \mathrm{Es}(-1.93), \mathrm{Ca}(-1.61), \mathrm{IP}(-1.58)$ & -0.12 \\
\hline 2001 & 5 & $\mathrm{~Pa}(-2.53), \mathrm{GP}(-2.24), \mathrm{GI}(-2.84), \mathrm{GS}(-2.17), \mathrm{Ca}(-2.33), \mathrm{IP}(-2.36)$ & -0.37 \\
\hline 2001 & 6 & $\mathrm{~Pa}(-2.76), \mathrm{GP}(-2.14), \mathrm{GI}(-2.49), \mathrm{GS}(-2.02), \mathrm{IP}(-2.09)$ & 0.02 \\
\hline 2001 & 7 & $\mathrm{~Pa}(-1.50), \mathrm{GP}(-2.62), \mathrm{GI}(-3.44), \mathrm{GS}(-1.90), \mathrm{Es}(-2.22), \mathrm{Ca}(-2.29), \mathrm{IP}(-2.19)$ & 0.11 \\
\hline 2002 & 5 & GP(-2.78), GI(-3.22), GS(-2.71), IP(-2.78), & 0.59 \\
\hline 2004 & 3 & $\mathrm{~Pa}(-2.31), \mathrm{GP}(-2.07), \mathrm{GI}(-2.19), \mathrm{GS}(-2.59), \mathrm{PB}(-2.12), \mathrm{Ro}(-2.02), \mathrm{Es}(-2.08), \mathrm{Ca}(-2.68), \mathrm{IP}(-2.29)$ & 0.09 \\
\hline 2004 & 4 & $\mathrm{~Pa}(-2.45), \mathrm{GI}(-2.16), \mathrm{GS}(-2.33), \mathrm{PB}(-2.88), \mathrm{Ro}(-3.51), \mathrm{Es}(-1.82), \mathrm{Ca}(-1.63), \mathrm{IP}(-1.76)$ & 0.11 \\
\hline 2004 & 5 & $\mathrm{~Pa}(-2.65), \mathrm{GP}(-2.38), \mathrm{GI}(-2.55), \mathrm{GS}(-2.04), \mathrm{PB}(-1.82), \mathrm{Ro}(-2.12), \mathrm{Ca}(-1.93), \mathrm{IP}(-2.26)$ & 0.05 \\
\hline 2005 & 10 & $\mathrm{Pal}(-2.02), \mathrm{GI}(-2.13), \mathrm{GS}(-1.88), \mathrm{PB}(-1.59), \mathrm{Ro}(-1.80), \mathrm{Es}(-2.01), \mathrm{Ca}(-1.66), \mathrm{IP}(-3.67)$ & -0.02 \\
\hline 2007 & 7 & $\mathrm{~Pa}(-1.61), \mathrm{GP}(-1.81), \mathrm{GI}(-2.00), \mathrm{Ro}(-2.30), \mathrm{IP}(-1.58)$ & 0.50 \\
\hline 2007 & 8 & GI(-1.66), Ro(-2.20), Es(-2.26), IP(-1.94) & 0.24 \\
\hline 2007 & 10 & GP(-2.16), GI(-1.54), Ro(-1.73), IP(-2.55) & 0.12 \\
\hline 2007 & 11 & $\mathrm{~Pa}(-1.64), \mathrm{GP}(-3.88), \mathrm{GI}(-1.66), \mathrm{GS}(-2.58), \mathrm{PB}(-2.27), \mathrm{Ro}(-2.44), \mathrm{Es}(-2.82)$ & -0.15 \\
\hline 2008 & 9 & GP(-1.58), GI(-1.87), GS(-1.77), Es(-1.58), IP(-1.54) & 0.59 \\
\hline 2009 & 1 & $\mathrm{GP}(-1.88), \mathrm{PB}(-3.25), \mathrm{Ro}(-3.57), \mathrm{Es}(-2.05)$ & -0.36 \\
\hline 2009 & 2 & $\mathrm{~Pa}(-2.22), \mathrm{GP}(-2.50), \mathrm{GI}(-2.40), \mathrm{GS}(-1.66), \mathrm{PB}(-2.92), \mathrm{Es}(-2.44)$ & -0.50 \\
\hline 2009 & 3 & $\mathrm{~Pa}(-2.58), \mathrm{GP}(-2.71), \mathrm{GI}(-2.71), \mathrm{PB}(-2.69), \mathrm{Es}(-2.61), \mathrm{IP}(-1.85)$ & -0.64 \\
\hline 2009 & 7 & $\mathrm{~Pa}(-1.84), \mathrm{GP}(-1.98), \mathrm{GS}(-1.51), \mathrm{IP}(-1.58)$ & 0.63 \\
\hline
\end{tabular}

alisio asociado a las anomalías positivas de la OAN (Hurrell 2005). Se observó del análisis realizado, que el ciclo diurno de los registros de TSSM (no mostrados) es muy pequeño en todos los sitios de medición en Bahía Culebra, por lo que la principal variabilidad de la TSSM está asociada al ciclo anual (Fig. 9).
Este resultado del Cuadro 6, también concuerda parcialmente con lo descrito por Alfaro y Lizano (2001), ya que estos autores encontraron que las anomalías positivas (negativas) en la TSM del Pacífico Norte en Costa Rica, tiende a ser precedido por anomalías positivas (negativas) en el índice Niño 3.4. 


\section{CUADRO 6}

Probabilidades empíricas condicionales para los eventos cálidos y fríos observados en Bahía Culebra, dada una anomalía positiva o negativa en los índices Niño 3.4 y OAN. Los valores entre paréntesis son las frecuencias absolutas de los eventos mostrados en los Cuadros 4 y 5 . El valor $\chi^{2}$ asociado a la tabla es de 12.96, con una significancia estadística de $\alpha=0.0047$

TABLE 6

Empirical conditional probabilities for the warm and cool events observed at Bahía Culebra, given a positive or negative anomaly in the indexes Niño 3.4 and NAO. Values in parenthesis are the absolute frequencies for the events showed in Tables 4 and 5. The $\chi^{2}$ value associated is 12.96, with a statistical significance of $\alpha=0.0047$

\begin{tabular}{|c|c|c|c|c|}
\hline & $\begin{array}{c}\text { Niño } 3.4>0 \\
\& \text { OAN }>0\end{array}$ & $\begin{array}{c}\text { Niño } 3.4<0 \\
\& \text { OAN }>0\end{array}$ & $\begin{array}{c}\text { Niño } 3.4>0 \\
\& \text { OAN }<0\end{array}$ & $\begin{array}{c}\text { Niño } 3.4<0 \\
\& \text { OAN }<0\end{array}$ \\
\hline Cálido & $0.21(3)$ & $0(0)$ & $0.58(8)$ & $0.21(3)$ \\
\hline Frío & $0.08(2)$ & $0.54(14)$ & $0.19(5)$ & $0.19(5)$ \\
\hline
\end{tabular}

Por otro lado, el hecho de que solamente el $54 \%$ de los eventos fríos coincidieron con anomalías negativas de la TSM en dicha región, podría estar asociado con que algunos eventos fríos observados en Bahía Culebra están relacionados con el paso de frentes fríos sobre el Mar Caribe, los cuales tienen una resolución temporal menor a la mensual (Alfaro \& Cortés 2011), además de que el efecto de enfriamiento podría estar localizado en la región de Bahía Culebra, asociado con los pasos en las cordilleras y no al resto de la región mostrada en la Figura 1. Este mismo tipo de discrepancias entre el comportamiento de los datos usados de TSM y los datos de la TSSM registrados en Bahía Culebra fue también encontrada por Jiménez et al. (2001). Nótese además del Cuadro 6 que el $54 \%$ de los eventos fríos se presentaron en concordancia con anomalías negativas en el Niño 3.4 y positivas en la OAN. Los resultados mostrados asociados a los ciclos anuales y diarios en Bahía Culebra deben ser interpretados bajo un escenario de cambio climático, asociado a un calentamiento ocurrido desde el año 1854, además coherente con lo observado globalmente y que su variabilidad climática está influenciada no sólo por aquella ligada a la de El Niño-Oscilación del Sur en el Pacífico Ecuatorial, sino también por influencias de tipo "puente" atmosférico relacionadas con la variabilidad en el Océano Atlántico.

\section{AGRADECIMIENTOS}

El estudio se realizó dentro del marco de los siguientes proyectos de la Vicerrectoría de Investigación de la Universidad de Costa Rica: 808-98-013, 808-B0-092, 805-B0-065, 805-A9532, 808-A9-180, 808-A9-070, 805-A8-606, 805-A7-002 у 808-B2-400, у CRN2050-IAI. El apoyo dado por Ecodesarrollo Papagayo ha sido invaluable para mantener este programa de monitoreo por todos estos años. Le agradecemos a todos los asistentes que han colaborado durante los años de este programa de monitoreo. $\mathrm{Al}$ Centro de Investigación en Ciencias del Mar y Limnología (CIMAR) de la Universidad de Costa Rica se le agradece el apoyo logístico, y al Instituto Costarricense de Turismo por el uso de las instalaciones en Playa Panamá, Bahía Culebra. También a Laura Zúñiga, Natalie Mora, Aarón Vega y Elsie Troyo por su apoyo en el procesamiento de la información y la base de datos. A Vilma Castro por el aporte y asistencia de la estación meteorológica automática localizada en Playa Panamá.

\section{RESUMEN}

Bahía Culebra, Golfo de Papagayo, Costa Rica es una región de afloramiento estacional. Para determinar la relación entre el clima y la variabilidad de la temperatura sub-superficial, se analizaron los registros de la temperatura sub-superficial del mar de nueve estaciones localizadas 
en la Bahía. El análisis permitió caracterizar su ciclo anual y explorar su relación con fuentes de variabilidad climática que influencian el clima regional para el periodo 19982010. Los resultados se contextualizaron usando además los datos de una estación meteorológica automática que funcionó en la bahía junto con el registro de la temperatura superficial del mar para una rejilla que cubre la región de $9-11^{\circ} \mathrm{N}, 85-87^{\circ} \mathrm{W}$, para el periodo $1854-2011$. Debido a la tendencia positiva encontrada en la región para la temperatura superficial del mar, se concluye que los resultados mostrados asociados a los ciclos anuales y diarios en Bahía Culebra deben ser interpretados bajo un escenario de cambio climático, asociado a un calentamiento ocurrido desde 1854, además coherente con lo observado globalmente, y que su variabilidad climática está influenciada no sólo por aquella ligada a la de El Niño-Oscilación del Sur, en el Pacífico Ecuatorial, sino también por influencias de tipo atmosférico relacionadas con la variabilidad en el Océano Atlántico, debido a que los eventos cálidos (fríos) en Bahía Culebra tienden a ocurrir en concordancia con anomalías positivas y negativas (negativas y positivas) de los índices Niño 3.4 y OAN, respectivamente.

Palabras clave: Bahía Culebra, Costa Rica, temperatura del mar, temperatura superficial del aire, climatología, variabilidad climática, ENOS, OAN, América Central, Golfo de Papagayo.

\section{REFERENCIAS}

Alfaro, E.J. 2000. Response of air surface temperatures over Central America to oceanic climate variability indices. Top. Meteor. Oceanogr. 7: 63-72.

Alfaro, E.J. 2002. Some characteristics of the annual precipitation cycle in Central America and their relationships with its surrounding tropical oceans. Top. Meteor. Oceanogr. 9: 88-103.

Alfaro, E.J. \& J. Cortés. 2011. Atmospheric forcing of cool subsurface water events in Bahía Culebra, Costa Rica. Sometido a la Rev. Biol. Trop., este volumen.

Alfaro, E.J. \& O.G. Lizano. 2001. Algunas relaciones entre las zonas de surgencia del Pacífico Centroamericano y los océanos Pacífico y Atlántico tropicales. Rev. Biol. Trop. 49 (Supl. 2): 185-193.

Alfaro, E., J. Soley \& D. Enfield.2003. Uso de una tabla de Contingencia para Aplicaciones Climáticas (Use of a Contingency Table for Climatic Applications), ISBN 9978-310-00-2. ESPOL / FUNDESPOL, Guayaquil, Ecuador. $51 \mathrm{p}$.

Amador, J.A. 2008. The Intra-Americas Sea Low-Level Jet: overview and future research, trends and directions in climate research. Ann. N.Y. Acad. Sci. 1146: 153-188.

Amador, J.A., E.J. Alfaro, O.G. Lizano \& V.O. Magaña. 2006. Atmospheric forcing in the Eastern Tropical Pacific: A review. Prog. Oceanogr. 69: 101-142.

Bindoff, N.L., J. Willebrand, V. Artale, A, Cazenave, J.M. Gregory, S. Gulev, K. Hanawa, C. Le Quéré, S. Levitus, Y. Nojiri, C.K. Shum, L.D. Talley \& A.S. Unnikrishnan. 2007. Observations: Oceanic Climate Change and Sea Level, p. 385-432. In S. Solomon, D. Qin, M. Manning, Z. Chen, M. Marquis, K.B. Averyt, M. Tignor \& H.L. Miller (eds.). Climate Change 2007: The Physical Science Basis. Contribution of Working Group I to the Fourth Assessment Report of the Intergovernmental Panel on Climate Change. Cambridge Univ. Press, Cambridge, United Kingdom and New York, NY, USA.

Hurrell, J.W. 2005. North Atlantic Oscillation, p. 536-539. In J.E. Oliver (ed.). Encyclopedia of World Climatology. Springer, Netherlands.

Jiménez, C., J. Cortés, A. León \& E. Ruiz. 2001. Coral bleaching and mortality associated with the1997-98 El Niño in an upwelling environment in the eastern Pacific (Gulf of Papagayo, Costa Rica). Bull. Mar. Sci. 69: 151-169.

Magaña, V., J.A. Amador \& S. Medina. 1999. The midsummer drought over Mexico and Central America. J. Climate 12: 1577-1588.

Muñoz, A.C., W. Fernández, J.A. Gutiérrez \& E. Zárate, 2002. Variación estacional del viento en Costa Rica y su relación con los regímenes de lluvia. Top. Meteor. Oceanog. 9: 1-13.

Smith, T.M., R.W. Reynolds, T.C. Peterson \& J. Lawrimore. 2008. Improvements to NOAA's Historical Merged Land-Ocean Surface Temperature Analysis (1880-2006). J. Climate 21: 2283-2296.

Soley, F.J. 1994. Suavizamiento de series cronológicas geofísicas con ruido blanco y rojo aditivo. Rev. Geof. 41: 33-58.

Taylor, M.A. \& E.J. Alfaro. 2005. Climate of Central America and the Caribbean, p 183-189. In J.E. Oliver (ed.). Encyclopedia of World Climatology. Springer, Netherlands.

Trenberth, K.E., P.D. Jones, P. Ambenje, R. Bojariu, D. Easterling, A. Klein Tank, D. Parker, F. Rahimzadeh, J.A. Renwick, M. Rusticucci, B. Soden \& P. Zhai. 2007. Observations: Surface and Atmospheric Climate Change, p. 235-336. In S. Solomon, D. Qin, M. Manning, Z. Chen, M. Marquis, K.B. Averyt, M. Tignor \& H.L. Miller (eds.). Climate Change 2007: 
The Physical Science Basis. Contribution of Working Group I to the Fourth Assessment Report of the Intergovernmental Panel on Climate Change. Cambridge Univ. Press, Cambridge, United Kingdom.

Wesson-Acuña, A. 2005. Wind Study for Bahía Manzanillo, Marina Papagayo, Golfo de Papagayo, Costa
Rica. Informe Técnico. WATERMARK S.A., San José, Costa Rica. 157 p.

Wesson-Vizcaíno, M. 2006. Local Wind Wave Design Conditions at Marina Papagayo, Golfo de Papagayo, Costa Rica. Informe Técnico. WATERMARK S.A., San José, Costa Rica. 67 p. 
